\title{
Vital signs for children at triage: A multicentre validation of the revised South African Triage Scale (SATS) for children
}

\author{
M Twomey, ${ }^{1}$ BSc, PhD; B Cheema, ${ }^{1} \mathrm{MB}$ ChB; H Buys,${ }^{2,3} \mathrm{MB}$ ChB, FCP (SA); K Cohen, ${ }^{1,4} \mathrm{MB}$ ChB, MMed EM (SA); A de Sa, ${ }^{5,6} \mathrm{MB}$ ChB, MCFP; \\ P Louw, ${ }^{1,7}$ MB ChB, MMed EM (SA); M Ismail, ${ }^{8,6}$ MB ChB, MFamMed, Dip HIV Man (SA); H Finlayson, ${ }^{9}$ MB ChB, FC (Paeds), Cert ID (Paeds); \\ C Cunningham, ${ }^{10}$ BSocSc Nurs, Dipl Crit Care; A Westwood, ${ }^{11,12}$ MB ChB, FCP (SA), MD \\ ${ }^{1}$ Division of Emergency Medicine, University of Cape Town, South Africa \\ ${ }^{2}$ Red Cross War Memorial Children's Hospital, Cape Town, South Africa \\ ${ }^{3}$ Department of Paediatrics and Child Health, Faculty of Health Sciences, University of Cape Town, South Africa \\ ${ }^{4}$ New Somerset Hospital, Cape Town, South Africa \\ ${ }^{5}$ Retreat Community Health Centre, Cape Town, South Africa \\ ${ }^{6}$ School of Public Health and Family Medicine, Faculty of Health Sciences, University of Cape Town, South Africa \\ ${ }^{7}$ Paarl Hospital, Paarl, Western Cape, South Africa \\ ${ }^{8}$ Woodstock Community Health Centre, Cape Town, South Africa \\ ${ }^{9}$ Department of Paediatrics and Child Health, Faculty of Medicine and Health Sciences, Stellenbosch University, Parow, Cape Town, South Africa \\ ${ }^{10}$ Milnerton Mediclinic, Cape Town, South Africa \\ ${ }^{11}$ ProvincialDepartment of Health, Western Cape, South Africa \\ ${ }^{12}$ Department of Paediatrics, Faculty of Health Sciences, University of Cape Town, South Africa
}

Corresponding author: M Twomey (micheletwomey@gmail.com)

\begin{abstract}
Objective. To validate a revised version of the paediatric South African Triage Scale (SATS) against admission as a reference standard and compare the sensitivity of triage using: (i) clinical discriminators; (ii) an age-appropriate physiological composite score; and (iii) a combination of both.

Methods. A prospective cohort study was undertaken validating the revised paediatric SATS against outcome markers of children at six emergency centres during a 2-month period in 2011. The primary outcome marker was the proportion of children admitted. Validity indicators including sensitivity (Se), specificity, positive predictive value and negative predictive value (NPV) were used to estimate the validity. Associated percentages for over-/under-triage were used to further assess practical application of the paediatric SATS.

Results. A total of 2014 children were included. The percentage of hospital admissions increased with an increase in the level of urgency from $5 \%$ in the non-urgent patients to $73 \%$ in the emergency patients. The data demonstrated that sensitivity increased substantially when using the SATS, which is a combination of clinical discriminators and the Triage Early Warning Score (TEWS) (Se 91.0\%, NPV 95.3\%), compared with use of clinical discriminators in isolation (Se 57.1\%, NPV 86.3\%) or the TEWS in isolation (Se 75.6\%, NPV 89.1\%).

Conclusion. The results of this study illustrate that the revised paediatric SATS is a safe and robust triage tool.
\end{abstract}

S Afr Med J 2013;103(5):304-308. DOI:10.7196/SAMJ.6877

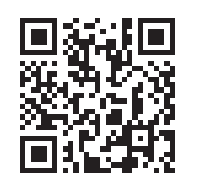

Emergency centre (EC) triage should enable rapid prioritisation of paediatric patients to determine how urgently children require care. Triage scales vary and are designed according to their unique context. ${ }^{[1]}$ Most are based on a list of clinical discriminators some include individual vital signs, while others include early warning scores (EWSs) or symptom-based algorithms.

When identifying life-threatening conditions in children, individual vital signs are known to be poor predictors. ${ }^{[2]}$ EWSs are known for their ability to detect physiological changes relating to vital signs ${ }^{[3]}$ Combining various standardised physiological parameters into an EWS has recently been recognised as a powerful tool in initiating appropriate responses from the initial contact at triage. ${ }^{[4,5]}$ The benefits of an EWS include its objectivity and the fact that an aggregated score is a stronger predictor than individual vital signs and reliance on routinely recorded vital signs. ${ }^{[6]}$
It is acknowledged that accurate triage is compromised when using EWSs without considering clinical discriminators such as purpuric rash and dehydration. ${ }^{[7]}$ In South Africa (SA), the Triage Early Warning Score (TEWS) - consisting of mobility, respiratory rate, pulse, temperature, level of consciousness and presence of trauma has been introduced as part of the South African Triage Scale (SATS), a multilayered approach to risk reduction at triage. Healthcare workers follow a simple algorithm (Appendix 1, available online: http://dx.doi.org/10.7196/SAMJ.6877) and perform triage based on clinical discriminators as well as an age-appropriate composite physiological score, the TEWS.

The SATS has four priority levels - emergency, very urgent, urgent and non-urgent, referred to as red, orange, yellow and green, respectively. The SATS has been used as an evidence-based triage tool in ECs across different levels of care in the Western Cape province of SA ${ }^{[8-13]}$ During 2011 the paediatric version of the SATS 
was revised (Appendix 1) and validated. This was carried out in response to a request by the Western Cape Department of Health, prompted by ongoing discussions and data that emerged from Red Cross War Memorial Children's Hospital. ${ }^{[14,15]}$ Two schools of thought had emerged among leaders around the most effective balance between using a clinical discriminators-based, more time-efficient triage tool as opposed to a combination of clinical discriminators and vital signs that takes a little longer. The recently published Red Cross study ${ }^{[14]}$ was not a comparison of an adapted triage tool (ETAT) and SATS, and therefore could not address the relative sensitivity of using clinical discriminators versus vital signs at triage.

The Paediatric Triage Working Group in the Western Cape province undertook a modified Delphi consensus process to revise the paediatric SATS and a sensitivity analysis to validate the revised tool. Consensus was reached on five major changes after several iterative rounds. These included revisions to: (i) add a clear step-by-step flowchart that makes explicit the first step of identifying emergency signs and asking for the presenting complaint; (ii) incorporate the $A B C c c D$ approach to identifying emergency signs from ETAT in the emergency clinical discriminators; (iii) ensure a four-level triage scale rather than a three-level scale with incorporation of ETAT priority signs and additional signs deemed necessary by the expert group; (iv) ensure that the step-by-step flowchart, clinical discriminators, TEWS and additional investigations are embedded in the wall chart for practical application; and $(v)$ develop a separate chart with additional tasks that do not change the priority level of the patient but are beneficial to patient care. The detailed revisions and rationale for changes are reported elsewhere (Western Cape Provincial Paediatric Triage Working Group, Revisions to the paediatric South African Triage Scale (SATS): Results of a modified consensus approach incorporating elements of ETAT). This report is being finalised for publication in a peer-reviewed journal.

This study aimed to validate the revised version of the paediatric SATS for children triaged in six different ECs and answer the question as to whether an age-appropriate TEWS in addition to clinical discriminators improves the sensitivity of identifying a sick child requiring admission versus using clinical discriminators or a TEWS independently.

\section{Methods \\ Study design}

This was a prospective observational design.

\section{Setting and sampling}

A convenience sample included six different ECs in the Western Cape. One hospital served the insured population in the private sector and the remaining 5 served the uninsured public sector ( 2 regional hospitals, 2 central hospitals, and 1 community health centre).

\section{Inclusion criteria}

All children younger than 13 years, presenting to triage as unscheduled patients, were included in the study.

\section{Method of measurement}

At each site one shift of nurses was trained to use the revised paediatric SATS. Convenience sampling included all children triaged by the nursing staff on that particular shift and lasted for 2 months from 3 October to 30 November 2011. Shifts included day, night and weekend periods. Information was collected on routine triage forms and later entered into a database. Data included presenting complaint, clinical discriminator, information on whether mobility was normal for age or not, respiratory rate, heart rate, temperature, level of consciousness on the AVPU scale (indicating whether the patient is A - alert, $\mathrm{V}$ - only responding to verbal stimulus, $\mathrm{P}$ - only responding to painful stimulus, $\mathrm{U}$ - unresponsive), and whether physical trauma was present or not. Triage information was prospectively documented by nursing staff. Disposition was documented retrospectively by the medical officer to obtain information on whether the patient was admitted or discharged within 24 hours. All patient information was made anonymous and entered into a password-protected computer to ensure patient confidentiality and privacy.

\section{Outcome marker}

In the absence of a gold standard for validation in triage, admission to hospital was used as a reference standard against which the paediatric SATS was validated. ${ }^{[1,17]}$

\section{Data analysis}

Validation was undertaken by calculating the sensitivity (Se), specificity $(\mathrm{Sp})$, associated under-triage (UT) and over-triage (OT) as well as negative predictive value (NPV) and positive predictive value (PPV) for the SATS, using admission and discharge as an outcome marker. The association between triage acuity level and proportions of admission was reported for acuity levels emergency, very urgent, urgent and routine. This analysis was undertaken by the Paediatric Triage Working Group in the Western Cape as part of a provincial validation process.

\section{Results}

During the validation period, a total of 2014 children were included. Their baseline demographics are presented in Table 1. The triage acuity level with the highest number of participants was the nonurgent category, representing $44.2 \%$ of the study population; the emergency category had the lowest number of participants (180 children), representing 9.9\%. A total of 443 children (21.5\%) were admitted and 1450 (72.0\%) were discharged.

Pooled data demonstrated admission proportions of $72.8 \%$, $29.0 \%, 27.9 \%$ and $4.7 \%$ for patients triaged into SATS acuity levels emergency, very urgent, urgent and non-urgent, respectively (Fig. 1).

Of the children, 1881 were included in the sensitivity analyses shown in Table 2 and Figs 2 - 4; 133 data forms were excluded, as 40 (2.1\%) had no documentation of disposition, $6(0.3 \%)$ were lacking

Table 1. Baseline characteristics of the participants

\begin{tabular}{ll}
\hline & Participants $(\mathbf{N = 2 ~ 0 1 4 )}$ \\
\hline Triage acuity level, $n$ (\%) & \\
Emergency (red) & $180(8.9)$ \\
Very urgent (orange) & $433(21.5)$ \\
Urgent (yellow) & $505(25.1)$ \\
Non-urgent (green) & $890(44.2)$ \\
Missing data & $6(0.3)$ \\
Disposition, $n$ (\%) & \\
Discharge & $1450(72.0)$ \\
Admission & $434(21.5)$ \\
Referral & $81(4.0)$ \\
Died & $1(0.1)$ \\
Missing data & $39(1.9)$
\end{tabular}




\section{RESEARCH}

information on the triage acuity level, $5(0.3 \%)$ had incomplete TEWS calculations, 1 patient died, and 81 patients (4.0\%) were referred and could not be followed up.

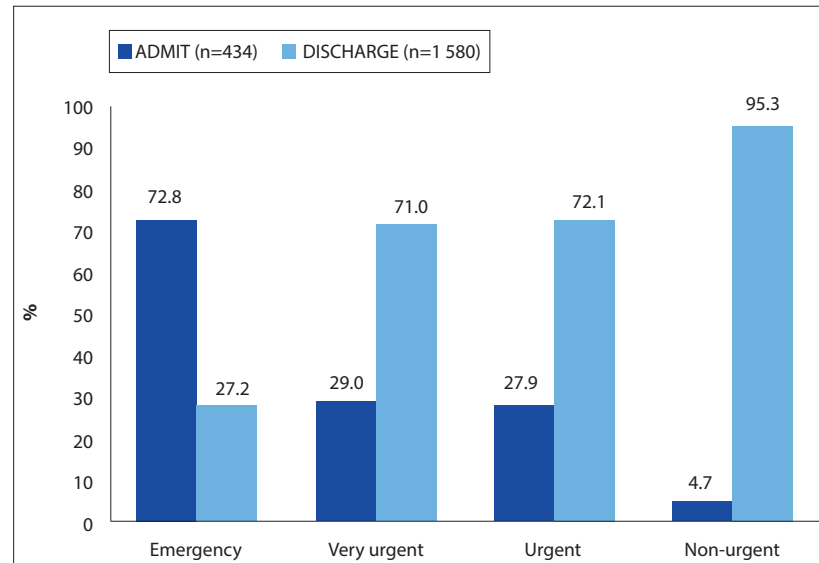

Fig. 1. Probability of admission and probability of discharge according to triage acuity level.

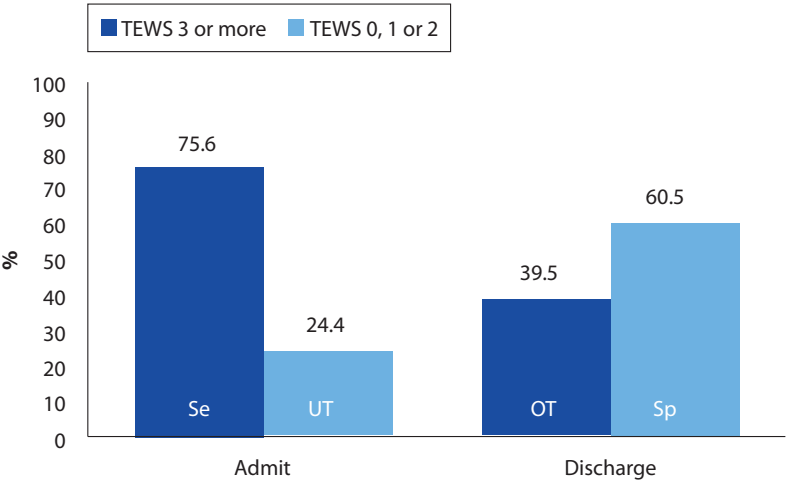

Fig. 3. Sensitivity (Se) with under-triage (UT) and specificity (Sp) with over-triage (OT) when using the Triage Early Warning Score in isolation.

\section{Discussion}

This validation study provides good evidence that the revised paediatric SATS is a robust and safe triage tool for children. There are no set standards for validation of triage tools, ${ }^{[16-18]}$ but it is sensible

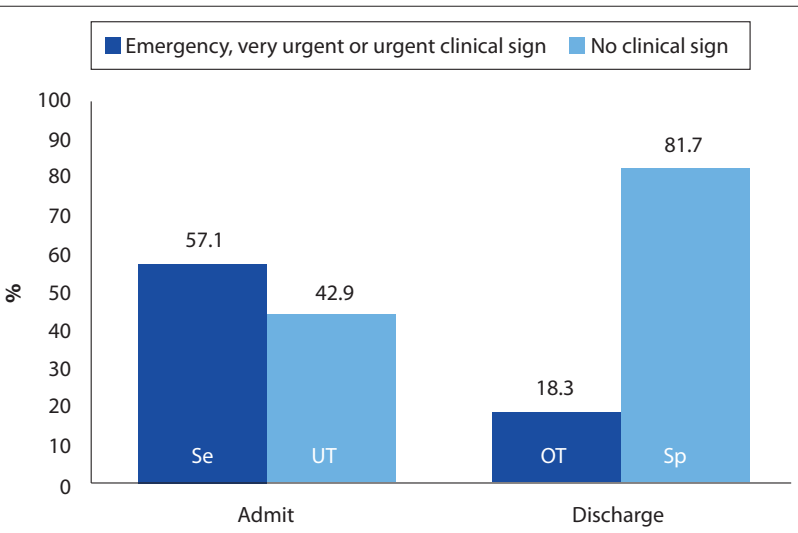

Fig. 2. Sensitivity (Se) with under-triage (UT) and specificity (Sp) with over-triage (OT) when using clinical discriminators in isolation.

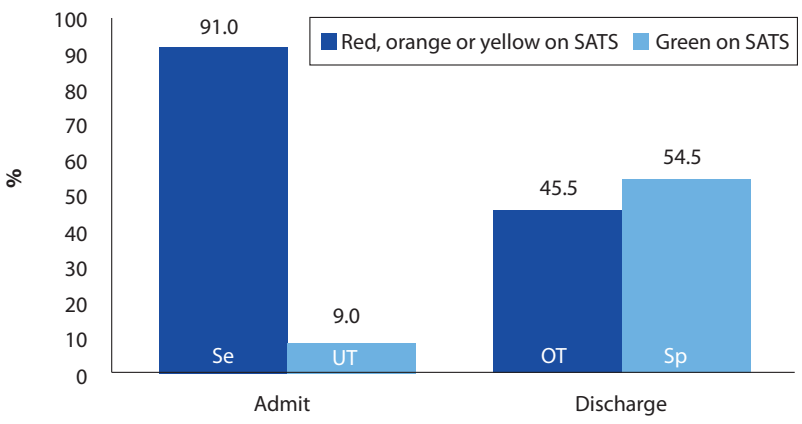

Fig. 4. Sensitivity (Se) with under-triage (UT) and specificity (Sp) with over-triage (OT) when using the South African Triage Scale (both clinical discriminators and Triage Early Warning Score).

Table 2. Comparing sensitivity, specificity, over-triage, under-triage, and positive and negative predictive values when using clinical signs or TEWS in isolation as opposed to in combination as the SATS

\begin{tabular}{|c|c|c|c|c|c|c|c|c|c|}
\hline & Admit & Discharge & Total & $\mathrm{Se}$ & Sp & OT & UT & PPV & NPV \\
\hline Using clinical signs in isolation & & & & 57.1 & 81.7 & 18.3 & 42.9 & 48.3 & 86.3 \\
\hline Emergency, very urgent, urgent sign & 248 & 265 & 513 & & & & & & \\
\hline No clinical sign & 186 & 1182 & 1369 & & & & & & \\
\hline Total & 434 & 1447 & 1881 & & & & & & \\
\hline Using TEWS in isolation & & & & 75.6 & 60.5 & 39.5 & 24.4 & 36.5 & 89.1 \\
\hline TEWS 3 or more & 328 & 571 & 899 & & & & & & \\
\hline TEWS 0,1 or 2 & 106 & 876 & 983 & & & & & & \\
\hline Total & 434 & 1447 & 1881 & & & & & & \\
\hline Using a combination of clinical signs and TEWS as SATS & & & & 91.0 & 54.5 & 45.5 & 9.0 & 37.5 & 95.3 \\
\hline SATS red, orange, yellow & 395 & 658 & 1053 & & & & & & \\
\hline SATS green & 39 & 789 & 828 & & & & & & \\
\hline Total & 434 & 1447 & 1881 & & & & & & \\
\hline
\end{tabular}


for tools to be deemed safer if they have high sensitivity and a high NPV (i.e. those children triaged to the lowest category are genuinely non-urgent cases). The paediatric SATS has been shown to have an Se of $91 \%$ and an NPV of $95.3 \%$, which demonstrates that it has an excellent ability to identify seriously ill children when admission is used as a marker for urgency.

Over-triage and under-triage are important concepts in triage. Over-triage is where triage is 'over-safe' and too many well children are incorrectly assigned to higher-priority categories. Under-triage is 'under-safe': ill children are incorrectly assigned to lower triage categories. In every triage tool there is a trade-off between over- and under-triage, with under-triage creating more risk of overlooking a sick child. Higher over-triage rates are therefore acceptable but need to be balanced against unnecessary use of limited resources. The paediatric SATS has an over-triage rate of $45.5 \%$; this is similar to the $37 \%$ reported using the Manchester triage tool. ${ }^{[19,20]}$ An overtriage rate of $16 \%$ was reported for the Emergency Severity Index, but this included parameters such as resource use in its evaluation and only defined non-admission of the sickest patients (ESI 1) as over-triage. ${ }^{[21]}$

Triage may be based on a variety of factors, including history, symptoms, clinical signs, vital signs and mobility. ${ }^{[22,23]}$ Often a combination of parameters is used. There is no consensus on the best method of triage for children. ${ }^{[7]}$ There is an ongoing debate regarding the value of vital signs in triage of children. ${ }^{[24]}$ Measuring vital signs in children can be problematic, especially in small or unco-operative infants, and furthermore normal ranges are not well defined for children. ${ }^{[6]}$ However, recent literature suggests that vital signs should be an integral part of safe triage. ${ }^{[23]}$ Our data strongly support use of the TEWS to augment clinical signs at triage.

This study found that triage on clinical signs alone had a sensitivity of $57.1 \%$, while triage based purely on the TEWS had a sensitivity of $75.6 \%$. Clearly, neither clinical signs alone nor vital signs alone make for an ideal triage tool, with under-triage rates of $42.9 \%$ and $24.4 \%$, respectively. This means that almost $43 \%$ of children who required admission had no clinical discriminator identified at triage. These children would have been triaged green (non-urgent) if a purely clinical signs approach had been used. Had triage been based purely on the TEWS, almost a quarter of children requiring admission would have been triaged green. However, the revised paediatric SATS (which uses a combination of clinical signs and the TEWS) resulted in $91 \%$ of the children requiring admission being appropriately prioritised.

A potential argument against incorporating vital signs into triage is the time taken to measure vital signs in children. The Emergency Nursing Association in the USA recommends that triage be achieved in $2-5$ minutes. ${ }^{[25]}$ There is little literature on the actual time taken for triage in emergency settings. One paediatric paper reported an average of 7 minutes at triage per patient. ${ }^{[24]}$ Another study, in a mixed emergency department, found a mean triage time of 2.4 minutes without vital sign measurement and 4.0 minutes with vital sign measurement, ${ }^{[25]}$ showing that taking of a full set of vital signs (blood pressure, pulse, respiratory rate and tympanic temperature) resulted in an additional 1.4 minutes per patient.

Measuring vital signs clearly adds to the time taken for triage. However, there is evidence that this additional time has safety benefits. The effect of vital signs on triage decision making was examined in 14285 patients triaged by 625 nurses at 24 hospitals. ${ }^{[2]}$ In 1050 children aged under 2 years, triage decisions were upgraded (increased in urgency) after measuring vital signs $8.7 \%$ of the time. Furthermore, $19 \%$ of triage decision upgrades were by two or more levels of urgency. The authors concluded that 'methods of triage that do not determine vital signs may not adequately reflect the urgency of patients' presentation. Further evidence comes from an increasing literature on the use of EWSs in paediatric patients, in which it has been shown that implementing physiological scoring systems to highlight potentially sick children has reduced serious preventable events such as respiratory and cardiac arrest. ${ }^{[27]}$

Children who are obviously unstable are triaged red (emergency) without vital sign measurement at triage in the paediatric SATS and most other triage systems. Paradoxically, it is the children who at first glance do not appear unwell who need the closest triage assessment to ensure correct prioritisation, especially as in SA public ECs patients can wait a very long time before being seen by a clinician. During this waiting time re-triage or nursing assessment is rarely performed. Furthermore, whereas in developed countries only trained and experienced nurses perform triage, this is not always the case in SA; 'acumen' and experience-based judgements on the severity of illness are therefore potentially less reliable. Given these realities, reliable safety nets are crucial to correctly identify any child who is 'subtly sick' and whose condition may deteriorate while waiting.

\section{Limitations}

Triage studies by their nature have limitations. A weakness of our study was that the reference standard used for validation was based on hospital admission alone. We were unable to assess EC length of stay or resource utilisation. In common with most triage studies, we were unable to follow up patients discharged from the EC. However, unlike many triage studies, which are often undertaken using 'paper cases' that are hypothetically triaged, our study has the strength of being a large-scale multicentre prospective study evaluating triage of real patients in ECs.

\section{Conclusion}

The revised paediatric SATS is a robust and safe triage tool for children. With an Se of $91 \%$ and an NPV of $95 \%$, it has excellent ability to identify potentially seriously ill children (using hospital admission as a marker for urgency). The integration of clinical discriminators with an age-appropriate physiological EWS at triage leads to a multilayered approach with multiple safety nets, resulting in a safer and more robust triage process, which is essential in a resource-constrained health setting such as ours. Further research is required on time taken to triage and alternative outcome measures in the SA context.

Acknowledgements. The authors thank all nursing and clinical staff who were trained and undertook triage during the 2 months of the study at Retreat Community Health Centre, Paarl Hospital, New Somerset Hospital, Red Cross War Memorial Children's Hospital, Tygerberg Hospital G Ground and Milnerton Mediclinic. We also thank Carol Kleinhans and Zanele Nxumalo and acknowledge the involvement of the entire Provincial Paediatric Triage Working Group.

\section{Competing interests. None.}

Contributors. MT conceived the idea, performed data analysis and wrote the first draft of the paper with BC; HB, KC, HF, PL, CC and MI undertook technical data collection; and all authors contributed to the final draft. 


\section{RESEARCH}

\section{References}

1. Iserson KV, Moskop JC. Triage in medicine. Part I: Concept, history, and types. Ann Emerg Med 2007;49(3):275-281. [http://dx.doi.org/10.1016/j.annemergmed.2006.05.019]

2. National Collaborating Centre for Women's and Children's Health. Feverish illness in children: Assessment and initial management in children younger than 5 years. Clinical Guideline CG47, National Institute for Health and Clinical Excellence, 2007. http://www.nice.org.uk/nicemedia/pd/ CG47Guidance.pdf (accessed 21 March 2013).

3. Subbe CP, Slater A, Menon D, Gemmell L. Validation of physiological scoring systems in the . emi.2006.035816]

4. Hancock A, Hulse C. Recognizing and responding to acute illness: Using early warning scores. British Journal of Midwifery 2009;17(2):111-117.

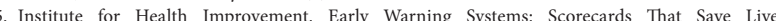

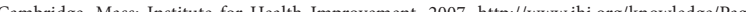
Cambridge, Mase he (2013).

6. Day A Oldroyd C. The use of early warning scores in the emergency department. J Emerg Nurs 2010;36(2):154-155. [http://dx.doi.org/10.1016/.j.jen.2009.11.001]

(1)

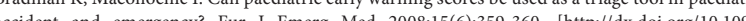

8. Gottschalk S, Wood D, DeVries S, et al., on behalf of the Cape Triage Group. The Cape Triage Score: A new triage system South Africa. Proposal from the Cape Triage Group. Emerg Med 2006:23(2):149-153. [http://dx doi org/10.1136/emj.2005.028332]

9. Bruijns SR, Wallis LA, Burch VC. A prospective evaluation of the Cape Triage Score in the emergency department of an urban public hospital in South Africa. Emerg Med J 2008;25(7):398402. [http://dx.doi.org/10.1136/emj.2007.051177]

0. Twomey M, Wallis LA. Workload and casemix in Cape Town emerency departments. S Afr Med 2007;97(12):1276-1280

11. Wallis LA. The Cape Triage Score: Update. Emerg Med J 2006;23:740. [http://dx.doi.org/10.1136 emj.2006.038778

2. Policy for Implementation of the Triage of Patients in Western Cape Emergency Centres. Circular H7 of 2006. Cape Town: Department of Health, Provincial Government, Western Cape, Sout Africa, 2006.

13. Twomey M, Wallis LA, Thompson ML, et al. The South African Triage Scale (adult version) provides valid acuity ratings when used by doctors and enrolled nursing assistants. African Journal of Emergency Medicine 2012;2(1):3-12. [http://dx.doi.org/10.1016/j.afjem.2011.08.014]
14. Buys H, Muloiwa R, Westwood C, Richardson D, Cheema B, Westwood A. An adapted triage tool (ETAT) at Red Cross War Memorial Children's Hospital Medical Emergency Unit, Cape Town: An evaluation. S Afr Med J 2013;103(3):161-165. [http://dx.doi.org/10.7196/SAMJ.6020]

15. Molyneux EM. Triaging children - keep it simple, swift and safe. S Afr Med J 2013;103(3):158-159. Molyneux EM. Truging (his:

16. Moll HA Challenges in the validation of triage systems at emergency departments. J Clin Epidemio 2010;63(4):384-388. [http:///dx.doi.org/10.1016/j.jclinepi.2009.07.009]

17. Twomey M, Wallis LA, Myers JE. Limitations in validating emergency department triage scales. Emerg Med J 2007;24(7):477-479. [http://dx.doi.org/10.1136/emj.2007.046383]

18. FitzGerald G, Jelinek GA, Scott D, Gerdtz MF. Emergency department triage revisited. Emerg Med J 2010;27(2):86-92. [http://dx.doi.org/10.1136/emj.2009.077081]

19. Van Veen M, Steyerberg EW, Ruige M, et al. Manchester triage system in paediatric emergency care Prospective observational study. BMJ 2008:337:a1501.

20. Van Veen M, Steyerberg EW, van't Klooster M, et al. The Manchester triage system: Improvements for paediatric emergency care. Emerg Med J 2012;29(8):654-659. [http://dx.doi.org/10.1136/ (11-200562]

21. Travers D, Waller AE, Katznelson J, Agans R. Reliability and validity of the Emergency Severity Index for pediatric triage. Acad Emerg Med 2009;16(9):843-849. [http://dx.doi.org/10.1111/j.1553 2712.2009.00494.x

22. McDaniel Hohenhaus S, Travers D, Mecham N. Pediatric triage: A review of emergency education literature. J Emerg Nurs 2008;34(4):308-313. [http://dx.doi.org/10.1016/j.jen.2007.06.022]

23. Fleming $\mathrm{S}$. Measurement and fusion of non-invasive vital signs for routine triage of acute paediatric illness. DPhil thesis, Oxford University, 2010. http://www.ibme.ox.ac.uk/bsp/publications/pdfiles/s_fleming_thesis.pdf (accessed 21 March 2013).

24. Keddington RK. A triage vital sign policy for a children's hospital emergency department. J Emer Nurs 1998;24(2):189-192. [http://dx.doi.org/10.1016/\$0099-1767(98)90030-7]

25. Travers D. Triage: How long does it take? How long should it take? J Emerg Nurs 1999;25(3):238 240. [http://dx.doi.org/10.1016/S0099-1767(99)70213-8]

26. Cooper R], Schriger DL, Flaherty HL, et al. Effect of vital signs on triage decisions. Ann Emerg Med 2002;39(3):223-232. [http://dx.doi.org/10.1067/mem.2002.121524]

7. Pearson G, Duncan H. Early warning systems for identifying sick children. Paediatrics and Child Health 2011;21(5):230-233. [http://dx.doi.org/10.1016/j.paed.2011.02.007

Accepted 21 March 2013 


\section{Appendix I}

Western Cape
covernment

Paediatric SATS Chart (5)

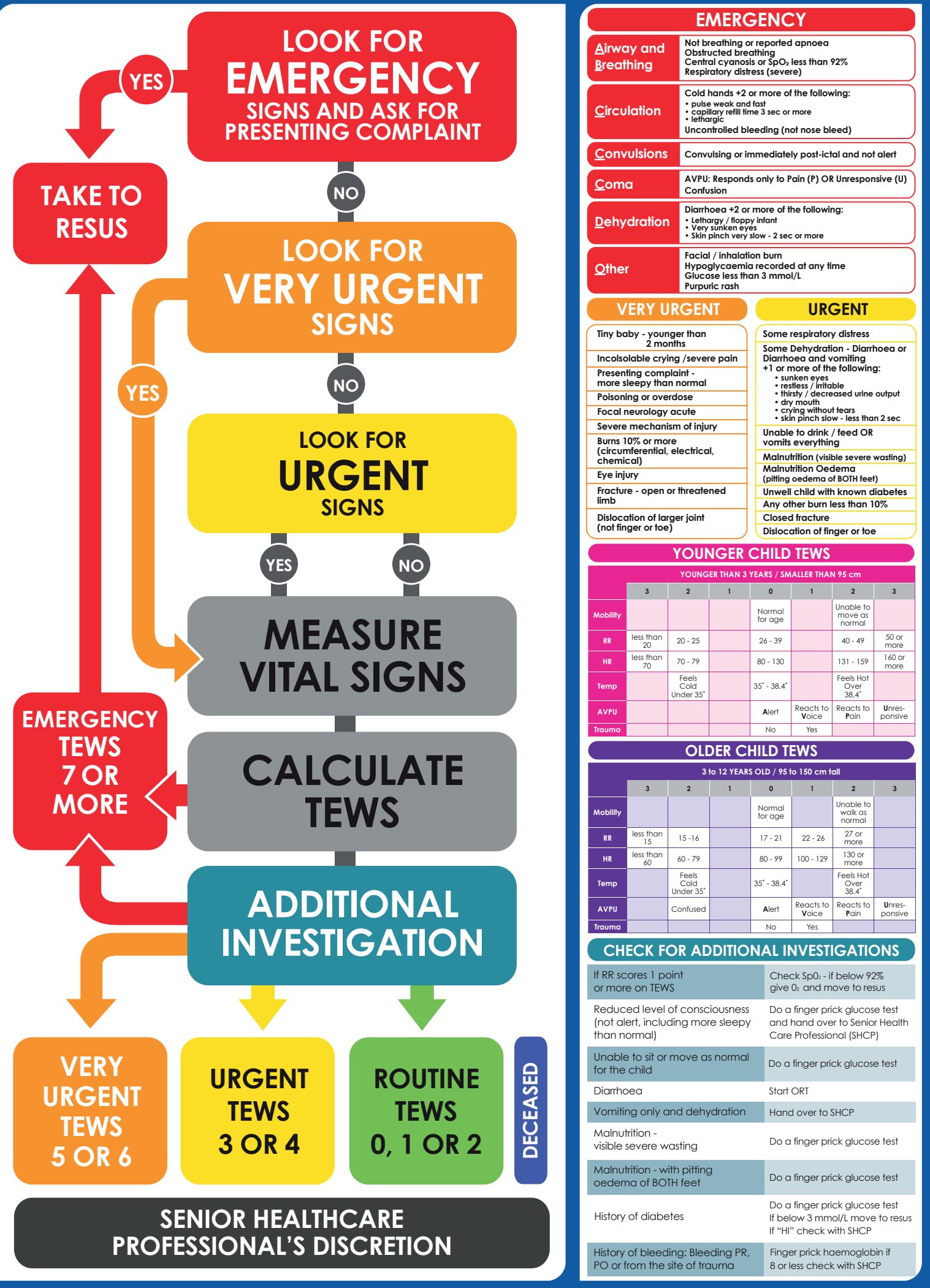

